\title{
Prediction of autobiographical memory interview ratings using quantitative text feature analysis.
}

\author{
Peters, J. ${ }^{1,2}$, Wiehler, A. ${ }^{* 1}$, Bromberg, U.*1 \\ ${ }^{*}$ equal contribution \\ ${ }^{1}$ Department of Systems Neuroscience, University Medical Center Hamburg- \\ Eppendorf, Hamburg, Germany. \\ 2Department of Psychology, Biological Psychology, University of Cologne, Albertus- \\ Magnus-Platz, Cologne, Germany.
}

Contact: jan.peters@uni-koeln.de

\begin{abstract}
Autobiographical memory and episodic future thinking (i.e. the capacity to project oneself into an imaginary future) are typically assessed using the Autobiographical Memory Interview (AMI). In the AMI, subjects are provided with verbal cues (i.e. "your wedding day") and are asked to freely imagine (or recall) the cued present or future situation. Narratives are then recorded, transcribed and analysed using and established manual rating procedure (Levine et al., 2002). Here we applied automatic text feature extraction methods to a relatively large $(n=86)$ set of AMI data. In a first proof-of-concept approach, we used regression models to predict manual AMI ratings from low-level linguistic features. Across a range of different regression methods, prediction accuracy averaged at about 0.5 standard deviations. Further analyses revealed that episodic and sematic detail ratings are association with both overlapping and distinct low-level linguistic features. Finally, we explored how prediction accuracy changes for test data sets of varying sizes. Prediction accuracy appeared to reach a maximum at about $n=3$ cues per condition, with additional test data failing to increase prediction accuracy. Our findings highlight the feasibility of using tools from quantitative text analysis to analyse AMI datasets, and we discuss potential future applications.
\end{abstract}




\section{Acknowledgements}

This research was supported by grants from the Deutsche Forschungsgemeinschaft (PE 1627/3-1, PE 1627/5-1). A.M., U.B. and J.P. designed research. A.M. and U.B. acquired the data. All authors analyzed the data and wrote the paper.

\section{Introduction}

Episodic autobiographical memory (AM) is central to our personal identity, and changes in this process characterize developmental phases as well as effects of neurological and psychiatric disorders. Recent findings have illustrated striking similarities in the neural systems supporting episodic memory and the capacity to mentally project oneself into the future (episodic future thinking, EFT) (Schacter et al., 2012). For example, AM and EFT are similarly affected in hippocampal amnesia (Hassabis, Kumaran, Vann, \& Maguire, 2007; Race, Keane, \& Verfaellie, 2011), Alzheimer's Disease (Addis, Sacchetti, Ally, Budson, \& Schacter, 2009; El Haj, Antoine, \& Kapogiannis, 2015), normal aging (Gaesser, Sacchetti, Addis, \& Schacter, 2011), semantic dementia (Irish, Addis, Hodges, \& Piguet, 2012; Viard et al., 2014) and traumatic brain injury (Rasmussen \& Berntsen, 2014), highlighting the close association between memory and future event construction (Hassabis \& Maguire, 2007; Schacter et al., 2012).

In addition, EFT impacts directly on other cognitive functions. For example, it has been speculated that EFT may facilitate future-oriented choice behaviour, i.e. behaviour that is advantageous only in the long-run (Boyer, 2008). One way to assess this type of behaviour is via temporal discounting (TD). In TD paradigms, the relative preference for smaller-sooner (SS) rewards over larger-but-later (LL) rewards is measured (Green \& Myerson, 2004; Peters \& Büchel, 2011). A stronger preference for SS rewards is taken as a measure of impulsivity, whereas a stronger preference for LL rewards is taken to reflect more future-oriented preferences. Boyer (2008) originally speculated that the ability to use EFT to project oneself into the future may help humans to override a natural tendency to make impulsive and short-sighted decisions, i.e. it may reduce the degree of temporal discounting. Recent years have brought forth increasing empirical support for the idea that EFT may, under certain conditions, modulate TD in this manner (Benoit, Gilbert, \& Burgess, 2011; Cheng, Shein, \& Chiou, 2012; Tinuke Oluyomi Daniel, Stanton, \& Epstein, 2013; T. O. 
Daniel, Stanton, \& Epstein, 2013; Lin \& Epstein, 2014; Palombo, Keane, \& Verfaellie, 2015; Peters \& Büchel, 2010). These interactions are particularly relevant for psychiatry, since TD is a reliable behavioural marker for a range of disorders of impulse control, including substance abuse and pathological gambling (Bickel, Koffarnus, Moody, \& Wilson, 2014). Therefore, understanding mechanisms of how TD can be reduced is of high clinical relevance.

Inter-individual differences in AM and EFT are typically assessed using variations of the autobiographical memory interview (AMI) (Levine, Svoboda, Hay, Winocur, \& Moscovitch, 2002). The AMI procedure involves exposing participants to a number of cues referring to future or past events, with the instruction to vividly recall $(A M)$ or imagine (EFT) these events. Participants verbally elaborate on their imaginations, which are recorded and transcribed. Transcripts are then manually rated using established procedures designed to dissociate e.g. episodic from semantic components (Levine et al., 2002). Thus, typical outcome measures of an AMI study include the number of episodic details (often termed internal details, as they pertain directly to the central event in question), the number of external details (episodic details not pertaining to the event in question) and semantic details (nonepisodic information). At present, the AMI can arguably be described as the "gold standard" in measuring AM and EFT. It is widely used and AMI ratings typically show high inter-rater reliability. There are, however, a few shortcomings of the procedure.

First, the multi-step procedure (interview, transcription, rating) it is very time consuming, and this might discourage researchers from using the AMI in studies with time contraints. Second, the ratings are subjective. For this reason, typically multiple independent raters rate at least a subset of the data, in order to ensure the reliability of the rating procedure. Finally, the rich and oftentimes very long narratives ${ }^{1}$ that participants produce during an AMI testing session contain a lot of linguistic information that could in principle be analyzed in a largely automatic fashion. However, by focussing mainly on AMI ratings, it is possible that potentially interesting information is ignored. The present study provides a first step towards a more automatic and quantitative analysis of linguistic content in AMI data by extracting lowlevel linguistic features from AMI narratives in a largely automatic fashion.

\footnotetext{
${ }^{1}$ The largest single subject AMI data set observed in one of our studies comprised 15 pages of single spaced text at font size 11 .
} 
We re-analysed a large set of AMI data $(n=86)$ using automatic extraction of low-level text features. Text features were computed both manually by crossreferencing words with publicly available linguistic data bases, and using commercially-available quantitative text analysis software, the Linguistic Inquiry and Word Count (LIWC) package (Pennebaker, Mehl, \& Niederhoffer, 2003). Note that one previous study applied the LIWC to autobiographical memory narratives (Schryer, Ross, St Jacques, Levine, \& Fernandes, 2012), but that study focused on differences in emotional content between younger and older adults. The first aim of this first report is a proof-of-concept, i.e. we aim to assess how well AMI ratings can be predicted from low-level linguistic features. To this end, we combined

dimensionality reduction and regression techniques with out-of-sample prediction (cross-validation). We then also explored whether different features are associated with e.g. internal vs. semantic details ratings. In the light of the known associations between EFT and temporal discounting (see above), we also used the same regression models to directly predict TD from linguistic features. Finally, we examined the degree to which prediction accuracy changes as a function of the amount of verbal material that is available for feature extraction.

\section{Methods}

\section{Participants}

We re-analyzed autobiographical memory interview data from two datasets. The first dataset (dataset 1) comprised interview data from $n=49$ adolescents (age 12-16, $X$ male). The second data set (dataset 2) comprised data from $n=20$ pathological gamblers (mean age [range]= [19-59] , 19 male), and from $n=20$ healthy control participants (mean age [range]= [18-58], 19 male). All subjects provided informed written consent prior to participantion. For minor participants, the parent or legal guardian provided written consent. The study procedures were approved by the local ethics committee (Hamburg Board of Physicians).

\section{Autobiographical Memory Interview}

Data were acquired using a modified version of the Autobiographical Memory Interview (AMI) (Levine et al., 2002). Interviews were conducted by U.B. (dataset 1) and A.W. (dataset 2) using a standardized protocol. For dataset 1, each participant was first instructed to report 12 personal episodic events ( 3 events within the next 6 
months, 3 events during the following school year, 3 events within the last 6 months and 3 events during the previous school year) from 3 different settings: 4 events related to family life, 4 events related to school life and 4 events related to their spare time activities). For dataset 2, each participant was instructed to report 5 personal episodic events that happened one year ago, and 5 personal episodic events that could happen one year from now. Verbal reports from both datasets were digitally recorded, transcribed, and then rated according to the original AMI manual (Levine et al., 2002).

\section{Temporal discounting}

Temporal discounting (TD) refers to the reward devaluation that typically occurs with increasing delay. All subjects completed a simple short and adaptive TD task (Peters \& Büchel 2009), that involved repeated choices between a smaller-sooner reward of $20 €$ available now and larger-but-later (LL) rewards available only after some delay $(2,7,14,30,90,180$ days). The procedure was adaptive such that the reward amount of the LL reward was increased following two successive choices of the SS reward, and decreased following two successive choices of the LL reward. Choice data were then fitted with a standard hyperbolic discounting function of the form $S V=A /(1+k D)$. Here, SV ist he subjective discounted value oft he reward, $\mathrm{A}$ ist he objective reward amount, $D$ is the delay in days, and $k$ is a subject-specific discounting function, where greater values reflect steeper discounting and thus more impulsive preferences. Fitting was performed using Maximum Likelihood Techniques implemented in Matlab (C) Version R2013a (The Mathworks). Details of the procedure are given elsewhere (Peters et al. 2012). As the resulting single-subject k-parameters are not normally distributed, we applied a square-root transformation prior to analyses (Ballard \& Knutson, 2009; Peters, Miedl, \& Büchel, 2012).

\section{Text feature extraction}

We used two largely complementary methods to extract low-level linguistic features from the narratives. First, we used custom in-lab Matlab (c) procedures to crossreference words with publicly available linguistic databases ('manual feature extraction'). Second, we used commercially available text analysis software ('Linguistic Inquiry and Word Count', LIWC) to obtain additional text features. The two approaches are described in detail in the following. 
Manual text feature extraction. Transcripts of narratives were first pre-processed to extract potentially relevant text features. The data from each subject were read into Matlab (C) and separated according to the two experimental conditions (EFT, AM). Then, individual words and sentences were extracted from the narratives, separately for each cue. Words were then further analyzed using Webservices provided by the "Projekt Deutscher Wortschatz" (http://wortschatz.uni-leipzig.de), which is part of the Leipzig Corpora Collection (REF?). Each word was converted to base form and classified (noun/adjective/verb/other) using the "baseform" Webservice (http://wortschatz.uni-leipzig.de/axis/servlet/ServiceOverviewServlet). For an input of e.g. freundlichste (nicest), this service returns both the baseform freundlich (nice) and the classification (adjective). This allowed us to calculate the proportion of nouns, verbs and adjectives separately for each condition. Next, we cross-referenced words (both pre- and post- baseform conversion) with the Berlin Affective Word List Reloaded (BAWL-R) (Vo et al., 2009). The BAWL-R is a database that contains $\approx 3000$ German words ( $\approx 2100$ nouns, $\approx 500$ verbs, $\approx 290$ adjectives). It provides normative ratings for the dimensions valence (-3 [very negative] through 0 [neutral] to +3 [very positive]), imageability (1 [hardly imageable] through 7 [highly imageable]), and emotional arousal (1 [low arousal] through 5 [high arousa/]) as well as a measure of word frequency (frequency/million). Figure 1 illustrates that baseform conversion increased the number of words that could be successfully cross-referenced with the BAWL-R database by around $50 \%$ in both experimental conditions. Based on the BAWL-R data, we calculated mean and variance of valence, arousal and imageability ratings for each subject and condition. Note that due to the highly skewed distribution of word frequencies, we used the median rather than the mean as a summary measure for each subject and condition. Based on these data, 19 predictors per experimental condition were obtained for each participant. The features are listed and explained in Table 1.

Linguistic Inquiry and Word Count (LIWC) features. We also applied a commercially available text analysis software that is frequently used in psychological research, the Linguistic Inquiry and Word Count (LIWC). LIWC is a dictionary-based method that counts words falling into one of roughly 80 content categories, and normalizes these counts by the total length of the texts. We used a validated German LIWC dictionary (Wolf et al. 2008) and separately analyzed EFT and AM data for each participant. 
Table 1. Labels, descriptions of manually-derived predictor variables (see 'manual feature extraction' section in the methods section). Each variable was computed separately for the EFT and AM conditions, yielding 32 predictors in total. BAWL-R - Berlin Affective Word List Reloaded (Vo et al., 2009).
Label
Description

\begin{tabular}{|c|c|}
\hline n_words & total number of words per condition (across all cues) \\
\hline n_sentences & total number of sentences per condtion (across all cues) \\
\hline total_classified_words & total number of words successfully classified as verb/adjective/noun \\
\hline n_adjectives & total number of words classified as adjectives \\
\hline n_verbs & total number of words classified as verbs \\
\hline n_nouns & total number of words classified as nouns \\
\hline p_adjectives & n_adjectives/n_words \\
\hline p_verbs & n_verbs / n_words \\
\hline p_nouns & n_nouns / n_words \\
\hline \multirow[t]{3}{*}{ n_bawl } & total number of words successfully cross-referenced with BAWL-R \\
\hline & database \\
\hline & post-baseform conversion \\
\hline \multirow[t]{3}{*}{ n_bawl_pre } & total number of words successfully cross-referenced with BAWL-R \\
\hline & database \\
\hline & pre-baseform conversion \\
\hline wps & words per sentence: $n \_w o r d s / n \_s e n t e n c e s$ \\
\hline m_emo & mean BAWL-R emotion rating \\
\hline m_arousal & mean BAWL-R arousal rating \\
\hline m_image & mean BAWL-R imageability rating \\
\hline v_emo & variance $B A W L-R$ emotion rating \\
\hline v_arousal & variance $B A W L-R$ arousal rating \\
\hline v_image & variance $B A W L-R$ imageability rating \\
\hline med_freq & median word frequency \\
\hline
\end{tabular}



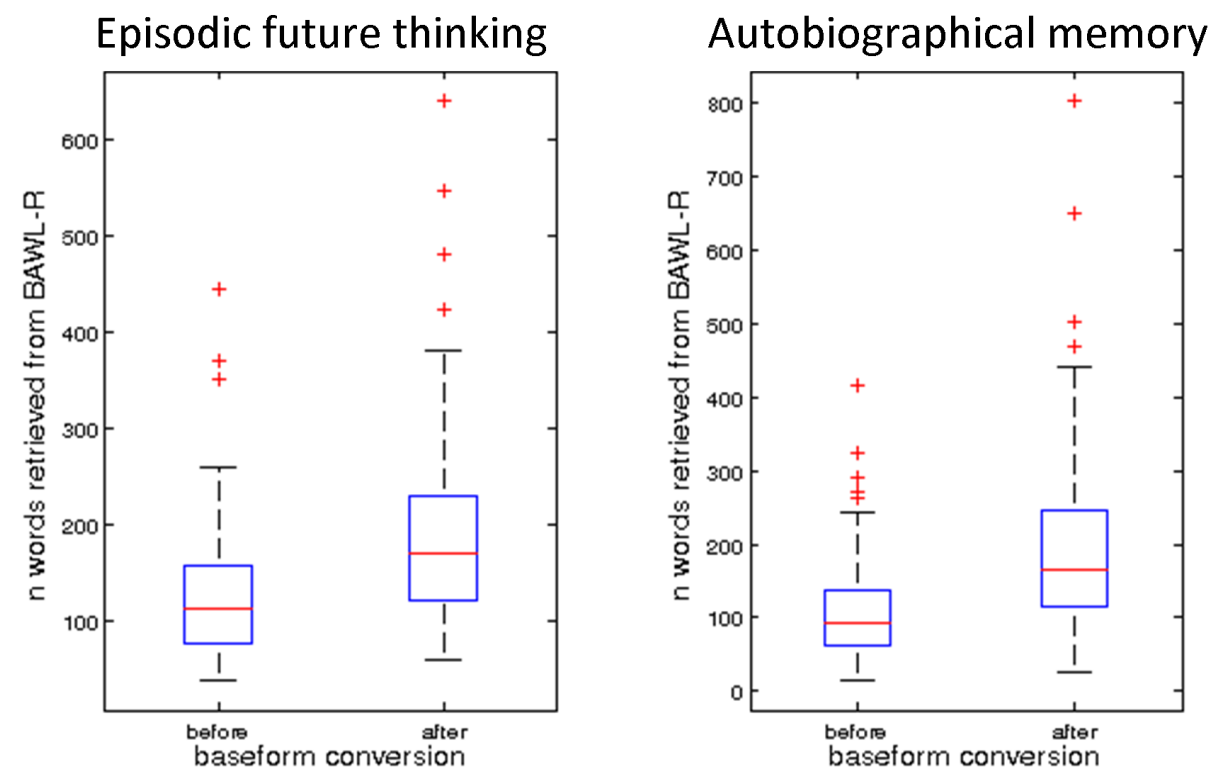

Figure 1. Baseform conversion increased the number of words successfully cross-referenced with the BAWL-R database (Vo et al., 2009) by around $50 \%$ for both EFT (left) and AM (right) conditions.

\section{Regression analyses}

To assess the association between text features (i.e. manual features, LIWC features) and $\mathrm{AMI}$ ratings we used regression techniques. Examination of the covariance structure of the predictor space revealed high co-linearity between some predictors (Figure 2). This poses a problem for standard multiple regression, as there is no unique least squares solution. We therefore applied regression techniques that can deal with co-linear data (Hastie, Tibshirani, \& Friedman, 2006).

Principal component regression (PCR). PCR consists of first performing a principal component analysis (PCA) on the data matrix $\mathbf{X}$. In the next step some target vector $\mathbf{y}$ is regressed onto a subset of $n$ of these components, with $n$ being typically determined via cross validation. PCA is a completely data-driven approach that extracts the main axes of variation from a multi-dimensional data set. Often, a relatively small subset of these principal directions accounts for the majority of variability in the data, and PCA is thus an effective technique for dimensionality reduction. The resulting component scores, which are linear combinations of the original variables, are orthogonal, and PCR thus solves the problem of predictor colinearity.

Partial least squares regression (PLS). PCR uses PCA to construct new orthogonal predictor variables, but it is completely data driven - components are constructed 
without regard to the target data $\mathbf{y}$ that one wishes to eventually predict. Thus it is possible that information predictive of $\mathbf{y}$ ends up in later components that are excluded from the PCR. Thus, when, the ultimate goal is prediction, PCR may not be the optimal choice. In contrast to PCA, in PLS, components are constructed based on both $\mathbf{X}$ and $\mathbf{y}$, that is, components are constructed such as to jointly maximize the amount of variance that is explained in $\mathbf{X}$ and the correlation of the resulting components with y (Hastie et al., 2006).

\section{Alternative regression approaches.}

For comparison with PCR and PLS, and to ensure that our results are independent of the regression approach adopted, we applied two additional techniques.

Supervised PCR (sPCR). Supervised PCR (Bair, Hastie, Paul, \& Tibshirani, 2006) is a recently proposed extension of standard PCR that involves an additional variable selection step. Columns of $\mathbf{X}$ that show little correlation with $\mathbf{y}$ are excluded before the PCA step of PCR. Which predictors are excluded is determined by the inclusion threshold $\theta$. The optimal value for $\theta$ is determined by cross-validation (see below).

Ridge regression. In ridge regression, the parameters of multiple linear regression are shrunk towards zero, with the degree of shrinkage being determined by the ridge tuning constant $\lambda$, which is determined by cross-validation. Shrinkage solves the problem of high variance in linear regression parameter estimates when predictors are highly correlated.

\section{Cross-validation}

We used leave-one-out (LOO) cross validation to quantify the out-of-sample prediction accuracy of all regression models, as well as to tune model hyperparameters (e.g. the shrinkage parameter $\lambda$ in ridge regression). To this end, models were fit to the data of all but one participant. We then calculated the root mean squared cross validation error across subjects as $R M S E=\sqrt{\frac{\sum_{i=1}^{n}\left(\hat{y}_{i}-y_{i}\right)^{2}}{n}}$ where $\hat{y}_{i}$ and $y_{i}$ are the predicted and actual data for the $i$-th subject. For the case of PCR and PLS, this procedure was repeated for a range of models with different numbers of included components in order to identify models maximizing out-of-sample prediction (i.e. minimizing over-fitting the training data). 

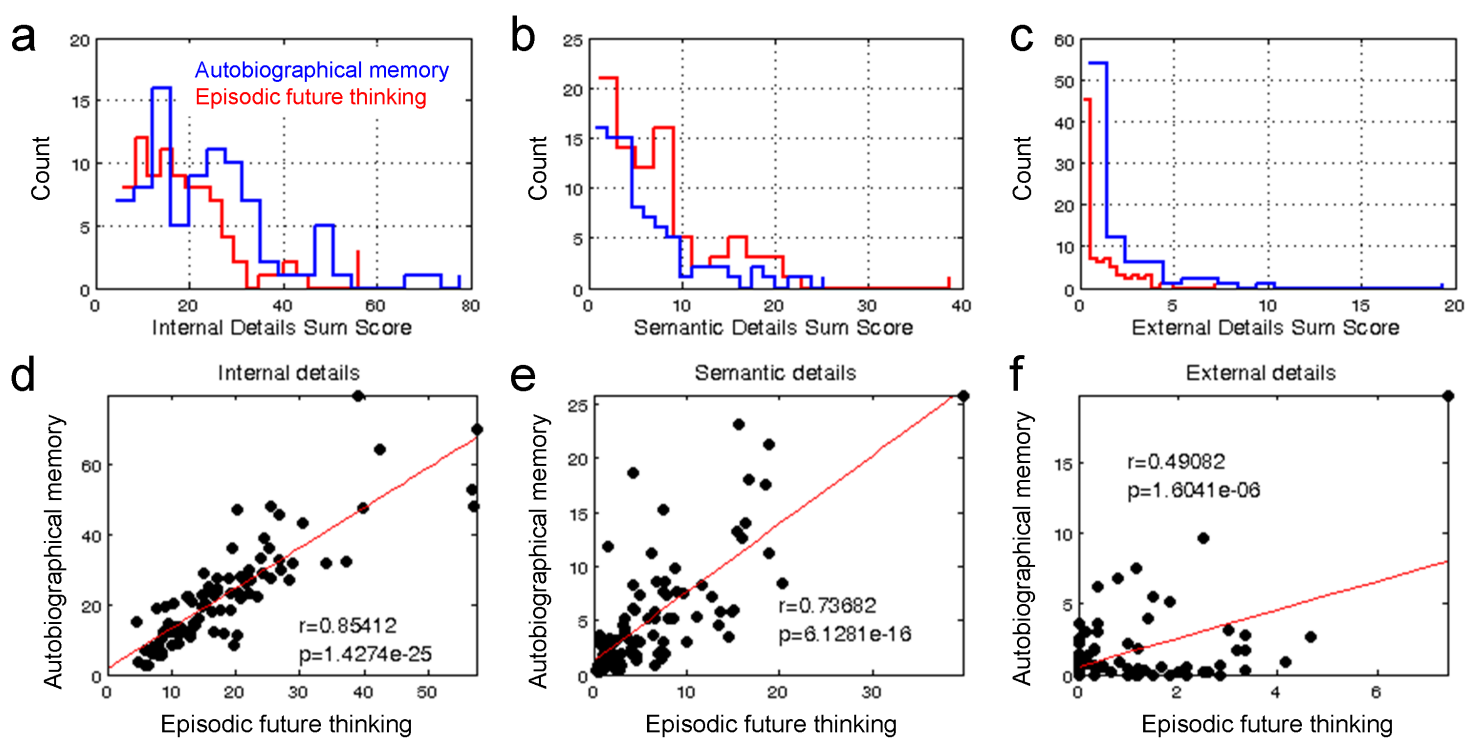

Episodic future thinking

Figure 2. Autobiographical memory interview data. Distributions of the ratings for each condition (blue - autobiographical memory [AM], red - episodic future thinking [EFT]) are shown in top row, and rating correlations between AM and EFT are shown in the bottom row. From left to right: internal episodic details, semantic details, external episodic details.

\section{Results}

\section{Autobiographical memory interview data}

Figure 2a-c shows histograms of the distributions of ratings for internal, semantic and external details. Overall, internal details ratings $\left(\mathrm{t}_{85}=5.0057, \mathrm{p}<0.001\right)$ and external details ratings ( $t_{85}=2.236, p=0.028$ ) where higher for $A M$ than $E F T$, whereas semantic details ratings were higher for $\mathrm{EFT}$ than $\mathrm{AM}\left(\mathrm{t}_{85}=2.5189, \mathrm{p}=0.014\right)$. All ratings were significantly correlated between conditions (Figure 2d-f).

\section{Text feature data}

We used two types of extracted text features (see methods section for details). First, text features were extracted using custom Matlab (c) code, in combination with research-dedicated word databases (see Table 1 for an overview of manual features). Second, we used commercially available software (Linguistic Inquiry and Word Count, see methods section) to derive dictionary-based word counts for 80 content categories. 


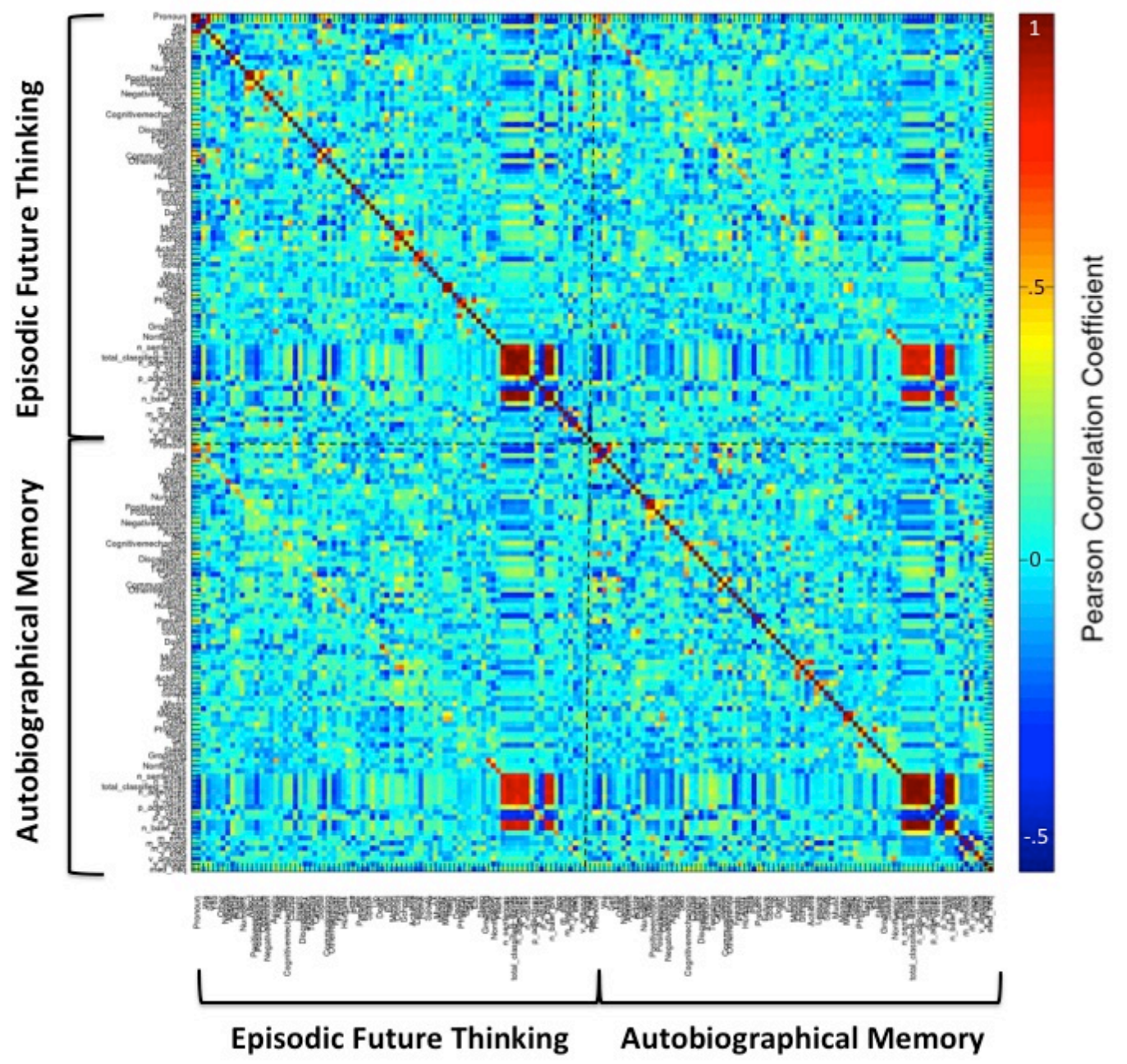

Figure 3. Pearson correlation matrix of all predictor variables, separated according to experimental condition (Autobiographical Memory [AM], Episodic Future Thinking [EFT]).

\section{Data correlation structure}

Figure 3 depicts the Pearson correlation matrix of the entire set of 160 predictor variables. Substantial collinearity between some variables is evident, in particular between the different variables measuring text quantity. Also, many measures showed considerable consistency across the two conditions (note the diagonal in the lower quadrant of the correlation matrix with generally positive correlation values). These correlations were highest for variables related to material quantity. But also, for example, the degree to which particular word classes were used (p_adjectives, $p_{-}$verbs, $p_{-}$nouns) as well as wps (words per sentence) and $m_{-}$image (mean BAWL imageability ratings) were positively correlated between conditions. Together, these findings suggest that both quantitative and qualitative 
aspects of the narratives were correlated between conditions. The correlation between manually derived text features and LIWC features was generally quite low, suggesting that the two types of features were not redundant.

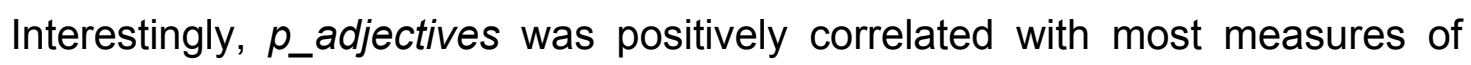
quantity, suggesting that the proportion of adjectives tended to increase with increasing lengths of the narratives - subjects producing longer narratives also incorporated a relatively greater number of adjectives in those narratives. Similarly, words-per-sentence (wps) was positively correlated with most measures of quantity, such that subjects producing longer narratives also produced relatively longer (and potentially more complex) sentences. 


\section{Principal Component}

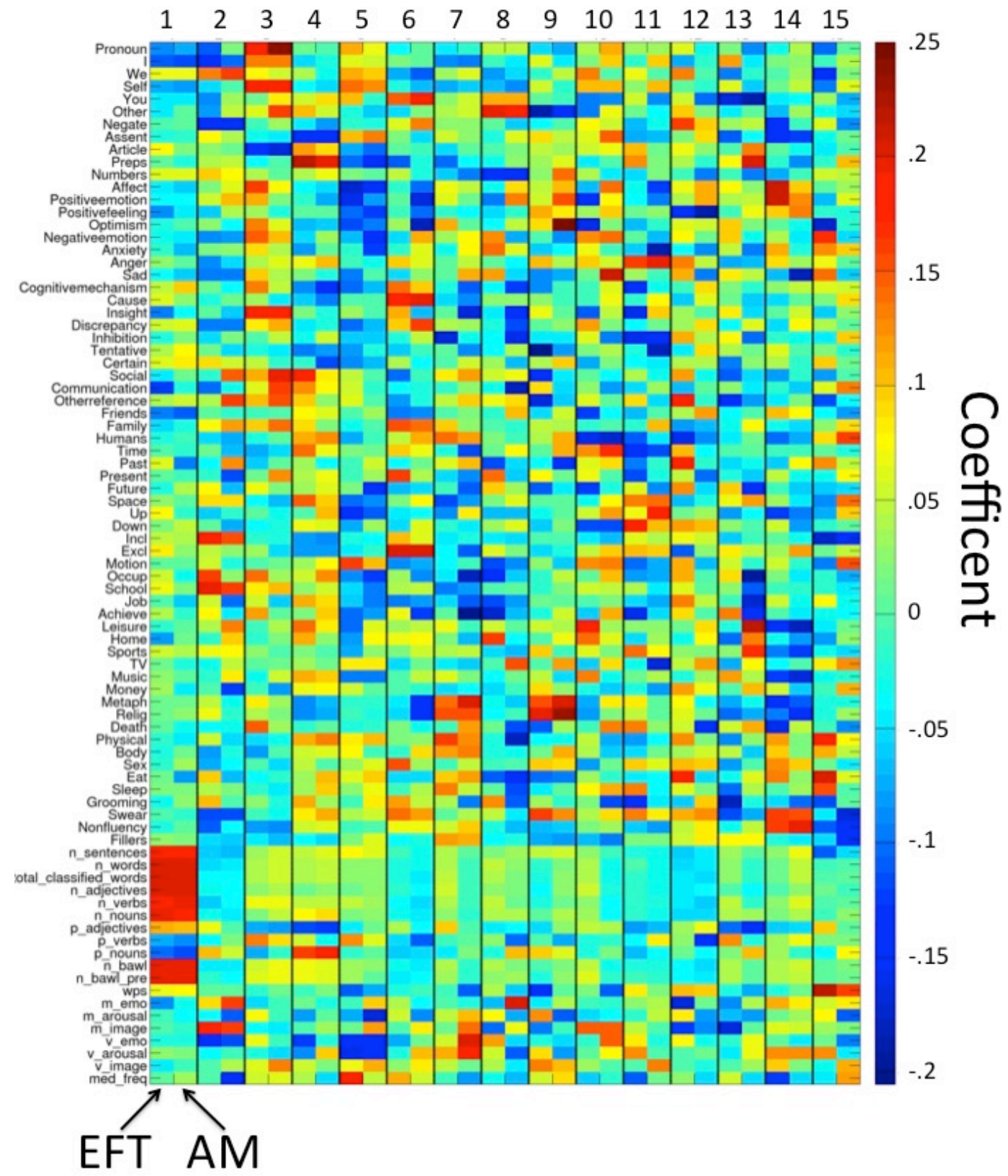

Figure 4. PCA coefficients per predictor, component and experimental condition. Note that coefficients for EFT and AM conditions are plotted next to each other to illustrate the similarity in loadings across conditions for the first few components.

\section{Principal component analysis and regression}

We next performed a principal component analysis (PCA) on the data to address the multi-collinearity problem (see Figure 1). Figure 4 illustrates predictor loadings of the first 10 principal components, and also illustrates the loading similarity between 
conditions. Similarity between conditions was most pronounced for the first few components, and decreased with increasing component number.
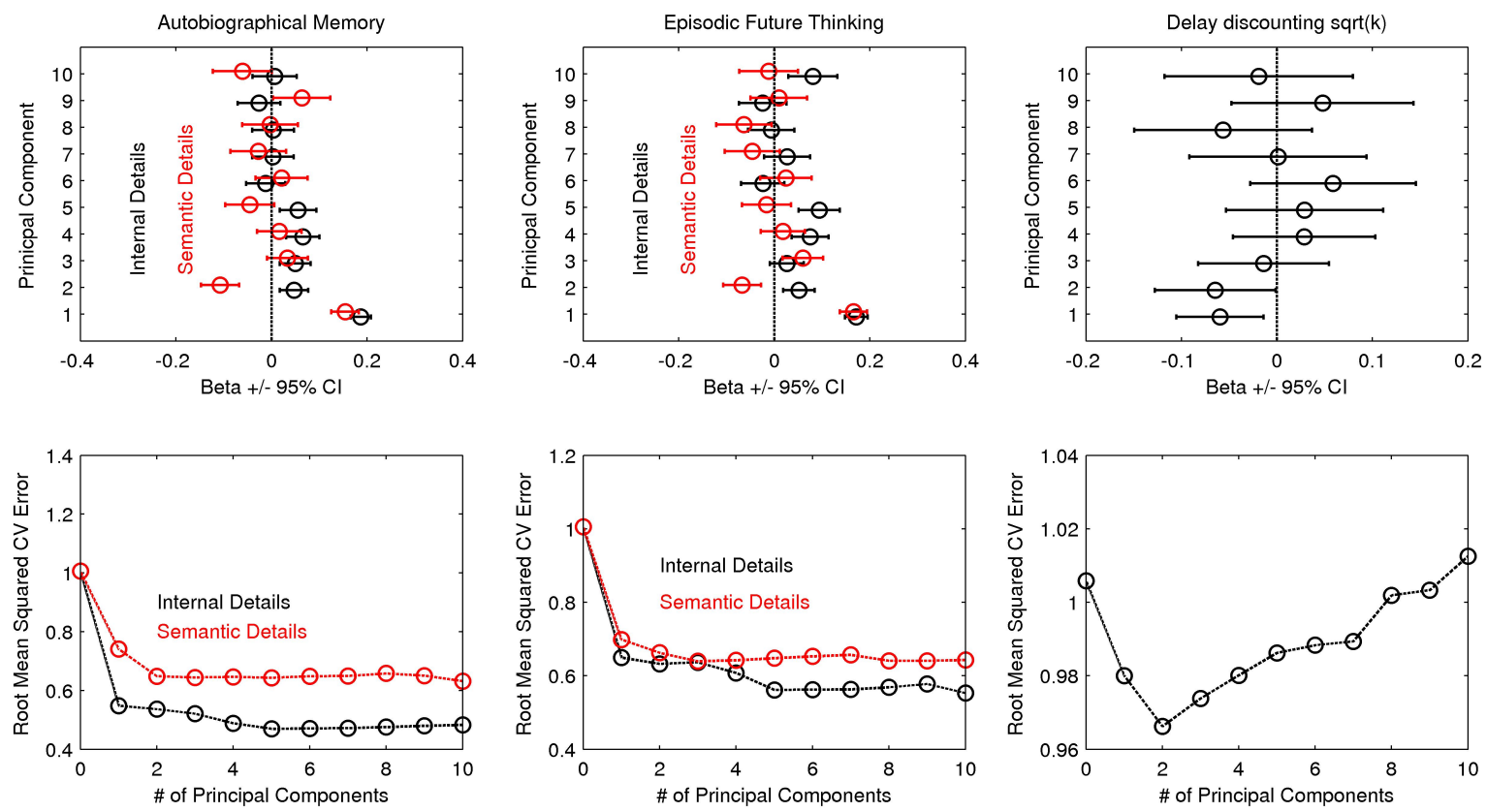

Figure 5. Regression coefficients (+/- 95\% confidence intervals) from a regression of internal (black) and semantic details ratings (red) onto the first ten principal components of text features (see Figure 4).

We next used principal component regression models to predict a range of different measures using the text feature data. In particular, we set up five analogous PCR models, all of which used the entire data matrix $\mathbf{X}$ for prediction. Model 1 and 2 predicted AM internal and semantic details ratings, respectively, models 3 and 4 predicted EFT internal and semantic details ratings, and model 5 predicted squareroot-transformed discount rates. As the PCA components used for prediction are solely based on the data, they were identical across models. This approach enabled us therefore to assess whether e.g. internal vs. semantic details ratings were associated with different text features. Figure 5 (top row) shows the regression coefficients for the first 10 PCs for each model. Internal and sematic details for AM and EFT were all positively and significantly (the $95 \% \mathrm{Cl}$ does not include 0 ) associated with the $1^{\text {st }} \mathrm{PC}$ (which predominantly reflects text quantity, see Figure 4). In contrast, e.g. the $2^{\text {nd }}$ and $5^{\text {th }}$ components (both reflecting qualitative aspects of the narratives, see Figure 4) were significantly positively associated with internal but not semantic details for both $\mathrm{AM}$ and EFT. PCR models with 5 components also 
produced the lowest cross validation error, i.e. best out-of-sample prediction (Figure $5)$.

Interestingly, the $1^{\text {st }}$ and $2^{\text {nd }} \mathrm{PCs}$ also showed a significant negative association with square-root transformed discount rates (95\% Cls for both regression coefficients were $<0$ ). Out of sample prediction, again using leave-one-out crossvalidation, revealed lowest prediction error for the two-component PCR model. However, overall prediction accuracy of discounting was only marginally better than the 0-component (intercept only) model.

\section{Alternative regression approaches}

For comparison, we repeated the prediction analyses of AMI ratings using 1) Ridge regression, 2) supervised PCR and 3) PLS (see methods for details). Best fitting hyper-parameters for these models (e.g. shrinkage parameter $\lambda$ for ridge regression, cut-off parameter $\theta$ for SPCR) where determined by LOO cross-validation, and are listed in Table 2. Differences between the different regression approaches were small (Hastie et al., 2006). RMSE for the best fitting model of each class is plotted in Figure 6.

Table 2. Hyper-parameters of the best-fitting regression models as determined by LOO crossvalidation ( $n$ - number of components included in the model, $\lambda$ - ridge shrinkage parameter, $\theta-\mathrm{sPCR}$ cut-off parameter).

\begin{tabular}{|c|c|c|c|c|c|}
\hline & PCR: & Ridge: & Super & PCR: & PLS: \\
\hline & $n$ & $\lambda$ & $\theta$ & $n$ & $n$ \\
\hline $\mathrm{EFT}_{\text {internal details }}$ & 5 & 80 & 46 & 9 & 2 \\
\hline $\mathrm{AM}_{\text {internal details }}$ & 5 & 62 & 51 & 4 & 3 \\
\hline Discounting sqrt(k) & 2 & 1168 & 51 & 4 & 1 \\
\hline
\end{tabular}




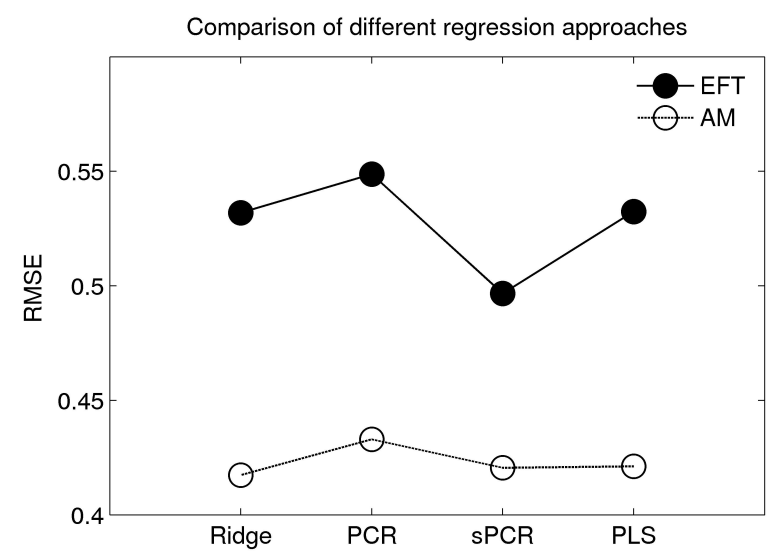

Figure 6. Comparison of regression techniques. Plotted are cross-validation errors (Root Mean Squared Error, RMSE) for four different techniques (Ridge - Ridge regression, PCR - Principal Component Regression, SPCR - supervised PCR, PLS - Partial Least Squares), predicting internal details ratings for EFT (black circles, solid lines) and AM (white circles, dashed lines). Prediction accuracy was very similar for the different approaches.

\section{Effects of text feature sets}

We next explored whether the LIWC and manually-derived text features performed differently in terms of prediction accuracy. To this end, we again set up regression models (PLS) to predict AM and EFT internal details ratings, but compared three analyses. Analysis 1 used only the LIWC features (and additionally included n_words, n_sentences, and words-per-sentence). Analysis 2 used only the Manual features (see Table 1). Analysis 3 combined all features used in analysis 1 and analysis 2. Prediction accuracy was best when the entire feature set was used for prediction (see Figure 7). For AM, prediction using manual features alone was better than using LIWC features alone, whereas the two feature sets performed similarly for EFT prediction. 

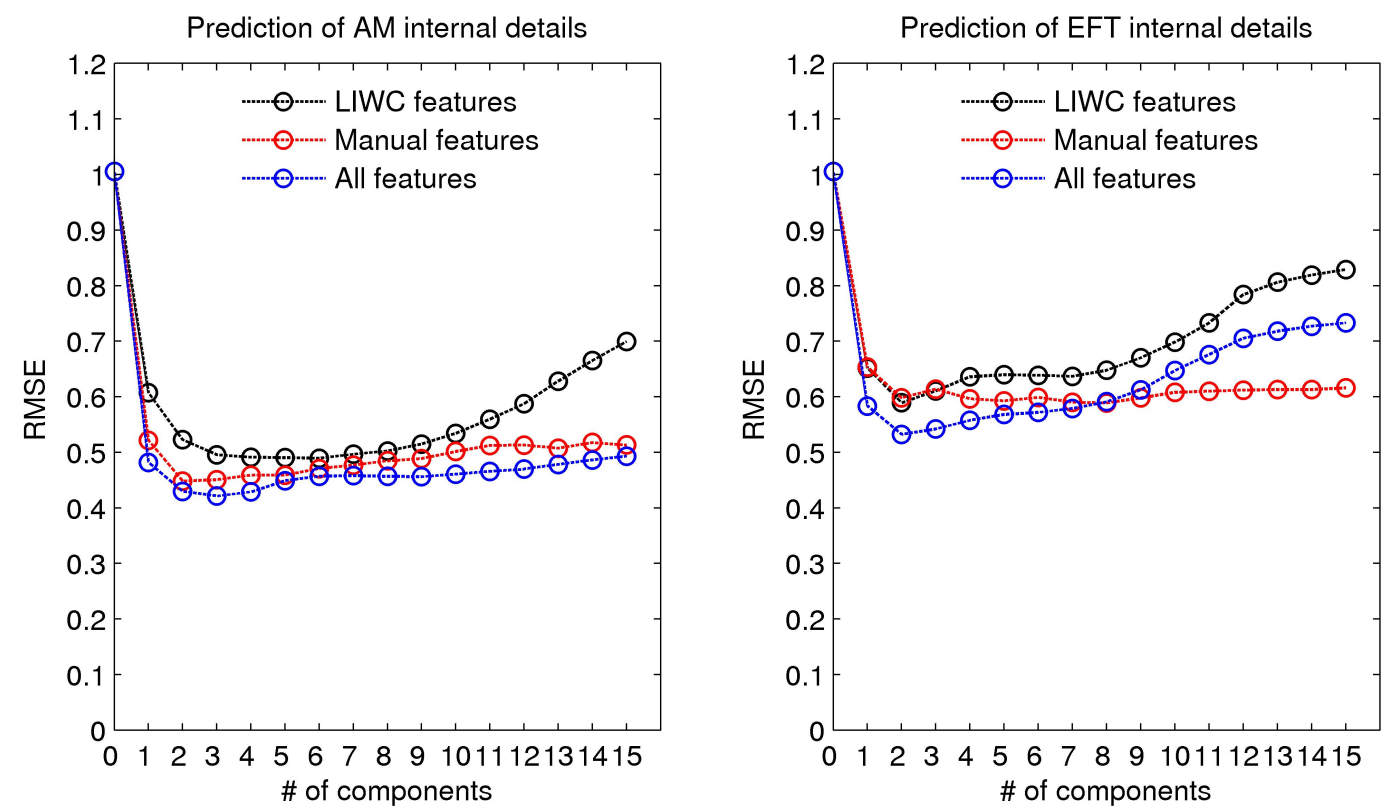

Figure 7. Effects of varying feature classes (black: only LIWC features, red: only manual features, blue: all features) on prediction accuracy (RMSE) using Partial Least Squares Regression models with different numbers of components. Prediction was best for the combined feature set, followed by the Manual features, and worst for LIWC features alone.

\section{Effects of test data amount}

Finally, we explored how reducing the amount of test data affects prediction accuracy. To this end, we systematically varied the amount of test data used for prediction (but not the amount of training data). Also, for simplicity, the following analyses used only the manual text features, and not the LIWC features. Given the similar results for the different regression approaches in the previous sections, we focussed on 2-component PLS models. Also, we used AM data for predicting AM ratings and EFT data to predict EFT ratings ${ }^{2}$. Thus, for each condition a separate 2component PLS model trained on the respective feature sets was used for prediction. We then varied the amount of test data used for prediction, to resemble a situation where one has a model trained on a large data set, but would like to more efficiently approximate AM/EFT in a subsequent test session. To this end, we first computed all manual features (see Table 1) separately for each cue (see methods section). Second, we fit a 2-component PLS model to the data from all but one subject (LOO cross-validation, see methods). Unlike the previous analyses, were we simply calculated the root mean squared CV error across subjects (see methods), we now

\footnotetext{
${ }^{2}$ This was done in order to more closely resemble a research situation where one might be particularly interested in just AM or EFT, and may thus only acquire a considerably smaller training data set by focussing on one of the two conditions
} 
averaged the CV error across all possible subsets of test data of a given size. Note that if there are $m$ cues per subject in total, then there are $\left(\begin{array}{l}j \\ m\end{array}\right)$ possible subsets of data from $j$ cues. For each subject $i$ and test data size $j$, we averaged the CV error across all test data subsets, and then computed the RMSE $\mathrm{RV}_{C V}$ across subjects, which is plotted in Figure 8. RMSE $C v$ decreased from 1 to 3 cues. For $\geq 3$ cues, no further improvement in prediction was observed. This was the case for both AM and EFT data. Note that overall, prediction accuracy was lower than in the previous PLS models, because in this analysis only condition-specific data were used. Accuracy was lower for EFT, likely because of the reduced amount of data available per cue in EFT vs. AM conditions. In terms of the original variables (internal details sum scores), this means that EFT rating predictions (based on EFT data) improved from being on average 9.50 points off (test data from 1 cue) to being on average 7.49 points off (test data from 3 or more cues). AM rating predictions (based on AM data) improved from being on average 11.55 points off (test data from 1 cue) to being on average 8.77 points off (test data from 3 or more cues).

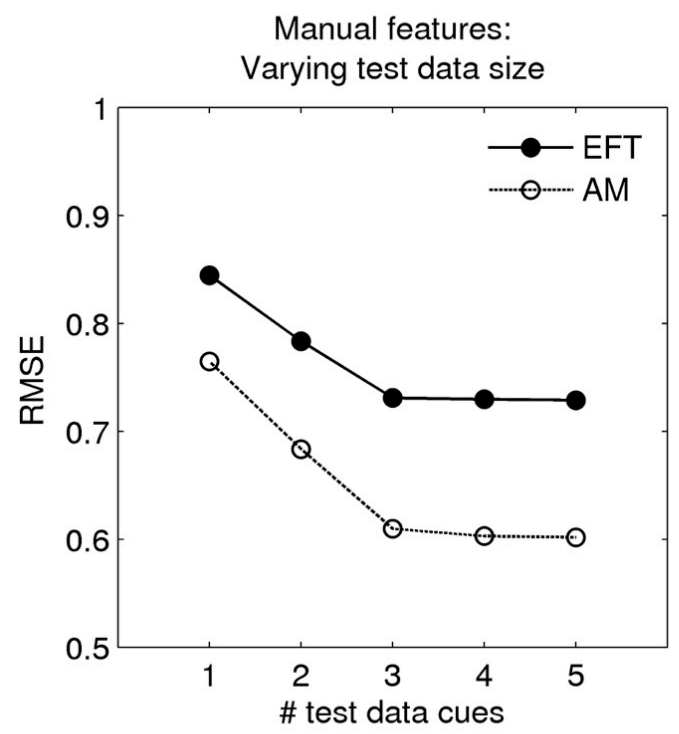

Figure 8. Effects of the amount of test data on prediction accuracy of a 2-component PLS model. The $\mathrm{X}$-axis depicts the number of event cues in the test data. The $\mathrm{Y}$-axis shows the cross-validation error for of AMI ratings (black: internal details EFT, red: internal details AM). Note: this analysis used the EFT text features to predict EFT ratings, and AM text features to predict AM ratings. Only manual features and not LIWC features (see methods) were used. 


\section{Discussion}

The ability to remember the past and to project oneself into the future is a core human cognitive capacity that is impaired in a range of psychiatric and neurological disorders. Memory and prospection processes are typically measured using variations of the Autobiographical Memory Interview (AMI), a procedure that combines verbal event elaboration with a manual rating procedure (Levine et al., 2002). Here we explore methods to analyse AMI data using automatic extraction of low-level linguistic features. In a first proof-of-concept approach, we applied regression techniques to predict standard manual AMI ratings from these features. Our findings suggest that $\mathrm{AMI}$ ratings can be predicted with reasonable accuracy from basic linguistic text features, with prediction accuracy averaging at about 0.5 standard deviations across analyses. Additional analyses show that different linguistic text features are associated with episodic and semantic detail ratings. Finally, we evaluated how changes in the amount of test data or the types of text features extracted from the texts affect prediction accuracy for manual ratings.

First, our analyses of a relatively large number of AMI interviews $(n=86)$ show that subject's event elaborations for past (AM) and future events (EFT) are correlated, not only in terms of the manual AMI ratings, but also in terms of lowerlevel text features: measures of both text quantity and quality were significantly correlated between conditions. In particular, the significant correlations of measures such as average word imageability ratings or word class proportions suggest that these features capture relevant between-subject variability. The results from principal component regression models used to predict internal and semantic details rating from AM and EFT revealed very similar patterns for the two experimental conditions. Taken together, these results support previous findings of a close association between AM and EFT (Schacter et al., 2012).

Second, results from the PCR show that generally, both episodic (internal) and semantic details scores from the AMI are strongly correlated with the raw quantity of verbal material produced by participants (first principal component). PCR revealed additional more qualitative components (e.g. the $2^{\text {nd }}$ principal component with high loadings on word imageability and valence) that where associated with episodic rather than semantic detail ratings. That is, use of more positive-valenced words and highly imageable words was correlated with greater episodic but not semantic details ratings. Taken together, these findings suggest that AMI ratings are do not solely 
capture variance due to narrative quantity, but also variance due to the quality of the elaborations. This supports previously reported dissociations between episodic and semantic memory and future thinking in different patient groups and age groups that have typically not reported group differences in narrative quantity (Addis et al., 2009; Addis, Wong, \& Schacter, 2008; Hassabis et al., 2007; Race et al., 2011). We show that although narrative quantity is associated with both internal and semantic details ratings, narrative quality, as measured by linguistic text features, independently contributes to variability in these AMI ratings.

For completeness, we compared a number of different regression approaches in terms of prediction accuracy. However, these analyses showed that the performance of the examined regression techniques was very similar. Of note, the relatively new approach of supervised principal component regression (sPCR) (Bair et al., 2006) performed quite well in particular for the EFT condition, which might be of interest for future studies. With respect to features, our findings suggest that the use of a combination of dictionary-based methods such as the LIWC and wordfeature methods (e.g. the manual feature extraction methods employed here) yielded the best prediction accuracy for AMI ratings. Future studies on quantitative text analysis might benefit from complementing dictionary-based methods such as the LIWC with additional text features such as those examined in the present study (e.g. proportions of different word classes).

Finally, by systematically varying the amount of test data used for prediction, we could show that increasing the test data size beyond $n=3$ cues per participant and condition does not further improve prediction accuracy. This might be of interest for future studies employing the methods described here for a semi-automatic analysis of new AMI data sets. However, it appears that there may be an upper limit for our feature-based prediction approach that is already reached with considerably smaller test data sizes than typically used in AMI research.

In the light of the known association between EFT and temporal discounting (Tinuke Oluyomi Daniel, Said, Stanton, \& Epstein, 2015; Tinuke Oluyomi Daniel et al., 2013; T. O. Daniel et al., 2013; Lin \& Epstein, 2014; Palombo et al., 2015; Peters \& Büchel, 2010), we also explored the extend to which TD can be directly predicted from AMI text features. Although the first two principal components from the text feature data were significantly associated with square-root-transformed discount rates (i.e. the $95 \%$ confidence intervals did not include 0 ), the out-of-sample 
prediction accuracy of a 2-component principal component regression model was quite low and only marginally better than an intercept-only model. Despite the transformation, the distribution of discount rates was still quite skewed, and this association was in part driven by a relatively small number of participants with high discount rates. We previously reported a reliable association between TD and EFT internal details ratings in the adolescent subsample of the present data set (Bromberg, Wiehler, \& Peters, n.d.). Although more data are clearly required, together, these findings suggest that TD may be more directly related to AMI ratings than the text feature data examined in the present study.

Although the vast majority of studies using the AMI were conducted in English speaking subjects, we analysed German AMI data. A validated LIWC dictionary was used (Wolf et al., 2008) and measure such as valence, arousal and imageability were extracted from a large published German word data base (Võ et al., 2009). A translation of the present approach to English language AMI data would therefore require additional programming efforts.

The present analyses constitute one of the first steps towards a more quantitative analysis of AMI data (Schryer et al., 2012). We focussed on exploring the association between the commonly used manual AMI ratings and automatically extracted text features. A number interesting research questions remain that were beyond the scope of this initial proof-of-concept study. First, we pooled data across three very different subject groups covering a large age range. Exploring how linguistic features vary as a function of e.g. age, clinical status or a range of other psychological constructs would be of considerable interest. Second, a comprehensive comparison of feature scores between AM and EFT was beyond the scope of the present paper, but might reveal interesting differences in how memory and future imaginations are processed. It would also be of considerable interest to further explore how such potential differences change e.g. across the life-span (Pennebaker \& Stone, 2003; Schryer et al., 2012) or as a result of neurological or psychiatric disease. 


\section{References}

Addis, D. R., Sacchetti, D. C., Ally, B. A., Budson, A. E., \& Schacter, D. L. (2009). Episodic simulation of future events is impaired in mild Alzheimer's disease. Neuropsychologia, 47, 2660-71.

Addis, D. R., Wong, A. T., \& Schacter, D. L. (2008). Age-related changes in the episodic simulation of future events. Psychol Sci, 19, 33-41.

Bair, E., Hastie, T., Paul, D., \& Tibshirani, R. (2006). Prediction by Supervised Principal Components. Journal of the American Statistical Association, 101(473), 119-137. http://doi.org/10.1198/016214505000000628

Ballard, K., \& Knutson, B. (2009). Dissociable neural representations of future reward magnitude and delay during temporal discounting. Neurolmage, 45, 143-50.

Benoit, R. G., Gilbert, S. J., \& Burgess, P. W. (2011). A neural mechanism mediating the impact of episodic prospection on farsighted decisions. J Neurosci, 31, 6771-9. Bickel, W. K., Koffarnus, M. N., Moody, L., \& Wilson, A. G. (2014). The behavioraland neuro-economic process of temporal discounting: A candidate behavioral marker of addiction. Neuropharmacology, 76, 518-527.

http://doi.org/10.1016/j.neuropharm.2013.06.013

Bromberg, U., Wiehler, A., \& Peters, J. (n.d.). Episodic future thinking is associated with impulsive decision-making in healthy adolescents. Child Dev.

Cheng, Y. Y., Shein, P. P., \& Chiou, W. B. (2012). Escaping the impulse to immediate gratification: the prospect concept promotes a future-oriented mindset, prompting an inclination towards delayed gratification. Br J Psychol, 103, 129-41. Daniel, T. O., Said, M., Stanton, C. M., \& Epstein, L. H. (2015). Episodic future thinking reduces delay discounting and energy intake in children. Eating Behaviors, 18, 20-24. http://doi.org/10.1016/j.eatbeh.2015.03.006

Daniel, T. O., Stanton, C. M., \& Epstein, L. H. (2013). The future is now: Comparing the effect of episodic future thinking on impulsivity in lean and obese individuals. Appetite, 71, 120-125. http://doi.org/10.1016/j.appet.2013.07.010 Daniel, T. O., Stanton, C. M., \& Epstein, L. H. (2013). The future is now: reducing impulsivity and energy intake using episodic future thinking. Psychol Sci, 24, 2339_ 42.

El Haj, M., Antoine, P., \& Kapogiannis, D. (2015). Similarity between remembering the past and imagining the future in Alzheimer's disease: Implication of episodic memory. Neuropsychologia, 66, 119-125. 
http://doi.org/10.1016/j.neuropsychologia.2014.11.015

Gaesser, B., Sacchetti, D. C., Addis, D. R., \& Schacter, D. L. (2011). Characterizing age-related changes in remembering the past and imagining the future. Psychol Aging, 26, 80-4.

Green, L., \& Myerson, J. (2004). A discounting framework for choice with delayed and probabilistic rewards. Psychol Bull, 130, 769-92.

Hassabis, D., Kumaran, D., Vann, S. D., \& Maguire, E. A. (2007). Patients with hippocampal amnesia cannot imagine new experiences. Proc Natl Acad Sci U S A, 104, 1726-31.

Hassabis, D., \& Maguire, E. A. (2007). Deconstructing episodic memory with construction. Trends Cogn Sci, 11, 299-306.

Hastie, T., Tibshirani, R., \& Friedman, J. (2006). The Elements of Statistical Learning: Data Mining, Inference and Prediction (Second Edition). Springer.

Irish, M., Addis, D. R., Hodges, J. R., \& Piguet, O. (2012). Exploring the content and quality of episodic future simulations in semantic dementia. Neuropsychologia, 50(14), 3488-3495. http://doi.org/10.1016/j.neuropsychologia.2012.09.012 Levine, B., Svoboda, E., Hay, J. F., Winocur, G., \& Moscovitch, M. (2002). Aging and autobiographical memory: dissociating episodic from semantic retrieval. Psychol Aging, 17, 677-89.

Lin, H., \& Epstein, L. H. (2014). Living in the moment: effects of time perspective and emotional valence of episodic thinking on delay discounting. Behavioral Neuroscience, 128(1), 12-19. http://doi.org/10.1037/a0035705

Palombo, D. J., Keane, M. M., \& Verfaellie, M. (2015). The medial temporal lobes are critical for reward-based decision making under conditions that promote episodic future thinking. Hippocampus, 25(3), 345-353. http://doi.org/10.1002/hipo.22376 Pennebaker, J. W., Mehl, M. R., \& Niederhoffer, K. G. (2003). Psychological Aspects of Natural Language Use: Our Words, Our Selves. Annual Review of Psychology, 54(1), 547-577. http://doi.org/10.1146/annurev.psych.54.101601.145041

Pennebaker, J. W., \& Stone, L. D. (2003). Words of wisdom: language use over the life span. Journal of Personality and Social Psychology, 85(2), 291-301.

Peters, J., \& Büchel, C. (2010). Episodic Future Thinking Reduces Reward Delay Discounting through an Enhancement of Prefrontal-Mediotemporal Interactions. Neuron, 66(1), 138-148. http://doi.org/10.1016/j.neuron.2010.03.026 Peters, J., \& Büchel, C. (2011). The neural mechanisms of inter-temporal decision- 
making: understanding variability. Trends in Cognitive Sciences, 15(5), 227-239. http://doi.org/10.1016/j.tics.2011.03.002

Peters, J., Miedl, S. F., \& Büchel, C. (2012). Formal Comparison of Dual-Parameter Temporal Discounting Models in Controls and Pathological Gamblers. PLoS ONE, 7(11), e47225. http://doi.org/10.1371/journal.pone.0047225

Race, E., Keane, M. M., \& Verfaellie, M. (2011). Medial temporal lobe damage causes deficits in episodic memory and episodic future thinking not attributable to deficits in narrative construction. The Journal of Neuroscience: The Official Journal of the Society for Neuroscience, 31(28), 10262-10269.

http://doi.org/10.1523/JNEUROSCI.1145-11.2011

Rasmussen, K. W., \& Berntsen, D. (2014). Autobiographical memory and episodic future thinking after moderate to severe traumatic brain injury. Journal of Neuropsychology, 8(1), 34-52. http://doi.org/10.1111/jnp.12003

Schacter, D. L., Addis, D. R., Hassabis, D., Martin, V. C., Spreng, R. N., \& Szpunar, K. K. (2012). The future of memory: remembering, imagining, and the brain. Neuron, 76, 677-94.

Schryer, E., Ross, M., St Jacques, P., Levine, B., \& Fernandes, M. (2012). Emotional expressivity in older and younger adults' descriptions of personal memories.

Experimental Aging Research, 38(4), 345-369.

http://doi.org/10.1080/0361073X.2012.699364

Viard, A., Piolino, P., Belliard, S., de La Sayette, V., Desgranges, B., \& Eustache, F. (2014). Episodic future thinking in semantic dementia: a cognitive and FMRI study. PloS One, 9(10), e111046. http://doi.org/10.1371/journal.pone.0111046 Võ, M. L.-H., Conrad, M., Kuchinke, L., Urton, K., Hofmann, M. J., \& Jacobs, A. M. (2009). The Berlin Affective Word List Reloaded (BAWL-R). Behavior Research Methods, 41(2), 534-538. http://doi.org/10.3758/BRM.41.2.534

Wolf, M., Horn, A. B., Mehl, M. R., Haug, S., Pennebaker, J. W., \& Kordy, H. (2008). Computergestützte quantitative Textanalyse. Äquivalenz und Robustheit der deutschen Version des Linguistic Inquiry and Word Count. Diagnostica, 54, 85-98. http://doi.org/10.1026/0012-1924.54.2.85 